\title{
OPINIÓN PÚBLICA Y PROPAGANDA: SU DEFINICIÓN, INTERPRETACIÓN Y SIGNIFICADO EN LOS ESTADOS UNIDOS DE LA PRIMERA POSGUERRA (1918-1922)'
}

\author{
Public opinion and propaganda: Their definition, \\ interpretation and meaning in the United States at the \\ beginning of the interwar period (1918-1922)
}

\author{
DARIO MIGLIUCCI \\ Universidad Complutense de Madrid \\ dariomigliucci@hotmail.com \\ Cómo citar/Citation \\ Migliucci, D. (2018) \\ Opinión pública y propaganda: su definición, interpretación \\ y significado en los Estados Unidos de la primera posguerra (1918-1922) \\ Historia y Política, 40, 213-238 \\ doi: https://doi.org/10.18042/hp.40.08
}

(Recepción: 04/06/2017. Evaluación: 29/09/2017. Aceptación: 10/01/2018. Publicación: 02/11/2018)

Resumen

El presente trabajo reconstruye la forma en la que los conceptos "propaganda» y «opinión pública» eran definidos y percibidos por los intelectuales y políticos norteamericanos en los primeros ańos después del fin de la Primera Guerra Mundial. Las fuentes documentales analizadas por este estudio fueron producidas por científicos sociales (obras académicas), periodistas (artículos de prensa) y representantes de las instituciones federales (discursos presidenciales) de la época. La tesis central de la investigación es que precisamente en el periodo que ha sido objeto de estudio se dio

Este trabajo ha sido desarrollado en el ámbito del programa FPU financiado por el Ministerio de Educación, Cultura y Deporte (FPU14/01884). 
un radical giro interpretativo en la valoración de las nociones «propaganda» y «opinión pública», debido sobre todo a las experiencias propagandísticas de la guerra, las frecuentes campañas de agitación de movimientos políticos radicales y la consolidación de nuevos y más eficaces medios de comunicación. Se defiende además que estas primeras reflexiones eruditas constituyeron el caldo de cultivo en el que se originó el célebre debate sobre construcción del consenso democrático que caracterizó el periodo de entreguerras.

\title{
Palabras clave
}

Propaganda; opinión pública; Estados Unidos; construcción del consenso; medios de comunicación.

\begin{abstract}
The present work reconstructs the way in which the concepts of 'propaganda' and 'public opinion' were defined and perceived by American intellectuals and policy-makers during the first years following the end of the Great War. The documentary sources analyzed for this study were produced by social scientists (academics works), journalists (press articles) y politicians (presidential speeches) of that time. The central thesis of the research is that, as for the interpretation of the notion of 'propaganda' and 'public opinion', a radical interpretative shift took place in that period as a consequence of the propagandistic experiences of the war, the recurrent radical movements' informative campaigns and the emergence of new and more efficient mass media. It is stated that these first scholarly postulates constituted the breeding ground for the emergence of the well-known debate on the manufacture of consent that characterized the interwar period.
\end{abstract}

\section{Keywords} media.

Propaganda; public opinion; United States; manufacture of consent; mass 


\section{SUMARIO}

I. INTRODUCCIÓN. II. EL CRECIENTE INTERÉS POR LA «PROPAGANDA» Y LA «OPINIÓN PÚBLICA». III. OPINIÓN PÚBLICA Y PROPAGANDA: INTENTOS DE CONCEPTUA. LIZACIÓN. IV. LA CREACIÓN DEL CONSENSO: UNA NUEVA HERRAMIENTA PARA LOS DIRIGENTES POLÍTICOS. V. CONCLUSIÓN. BIBLIOGRAFIA.

\section{INTRODUCCIÓN}

Durante el periodo de entreguerras se desarrolló en los Estados Unidos de América un encendido debate intelectual sobre la controvertida temática de la propaganda gubernamental. Por una parte, se cuestionó el hecho de que un régimen democrático estuviese legitimado para llevar a cabo actividades consagradas a la manipulación de las percepciones y actitudes de la opinión pública interna, y por otra, se barajó la posibilidad de que el Gobierno federal pudiese poner en marcha campañas de persuasión de la ciudadanía con el fin de contrarrestar la propaganda de naciones hostiles y movimientos políticos subversivos. Dicho debate fue protagonizado por numerosos exponentes del mundo de la cultura de la época, entre los cuales destacaron prestigiosos académicos como John Dewey y Harold D. Lasswell, célebres periodistas como Walter Lippmann, y famosos expertos en relaciones pública como Edward L. Bernays. El debate acabó abarcando toda una serie de cuestiones relacionadas con el control de la información, como la definición misma del concepto de propaganda, las campañas de manipulación de la Primera Guerra Mundial o la centralidad de la opinión pública dentro del sistema democrático².

A lo largo de las últimas décadas han sido numerosos los especialistas de distintas disciplinas que han abordado el análisis de la propaganda y de la opinión pública en sus diferentes aspectos. Un destacado número de autores se ha centrado en su conceptualización, matizándose —en el caso de la propaganda - las heterogéneas connotaciones - manipulación vs. persuasión vs. control de las masas - que pueden atribuirse a este complejo fenómeno, a partir sobre todo de la intencionalidad y los propósitos del propagandista ${ }^{3}$.

2 Algunas de las obras más destacadas que se produjeron en dicho contexto fueron Bernays (1923); Dewey (1927); Irwin (1936); Lasswell (1927); Lasswell (1941); Lee (1925); Lippmann (1922); Ponsonby (1928), y Read (1941).

3 Black (2001); Cunningham (2002), y Jowett y O’Donnell (2014). Estos últimos aseguran que la propaganda sirve deliberadamente al interés del propagandista 
También han sido estudiados significado, historia y facultades de la opinión pública, examinándose tanto su poder de agencia como su inevitable predisposición a ser moldeada por actores políticos, entidades económicas y factores culturales $^{4}$. Diversos investigadores, además, han explorado el proceso propagandístico en sus diferentes fases, desde la producción de políticas públicas a nivel estatal hasta la recepción de las mismas por parte de la opinión pública, pasando por la influencia o control que poder político y financiero ejercen sobre los medios de comunicación 5 . Una mención particular la merece la obra Opinión Pública, de Juan Ignacio Rospir, dedicada al caso norteamericano, en la que se analiza no solo la cuestión de la definición de la noción «opinión pública», sino también el complejo proceso —iniciado precisamente en Estados Unidos durante el periodo de entreguerras- de su medición científica mediante el elaborado sistema de las encuestas de opinión ${ }^{6}$. Finalmente, en relación con el debate sobre propaganda del periodo de entreguerras, recientemente diversos investigadores han analizado las distintas posturas defendidas en aquellos años por diferentes miembros del mundo de la cultura norteamericana, publicándose libros académicos y artículos científicos cuya finalidad ha sido la reconstrucción de la labor intelectual que fue llevada a cabo en aquellos años?

En cuanto al propósito del presente trabajo, se trata de reconstruir la forma en la que conceptos como «propaganda» $\mathrm{y}$ «opinión pública» eran percibidos en Estados Unidos en los años inmediatamente anteriores al comienzo de dicho debate. ¿Qué entendían por «propaganda» los dirigentes nacionales, los responsables de los medios de comunicación y los científicos sociales que protagonizaban la escena pública en los primeros años de la posguerra? ¿A qué se referían los distintos miembros del mundo político y cultural de aquella época cuando empleaban la polisémica expresión «opinión pública»? Lo cierto

(dañando, si es necesario para tal fin, al receptor), mientras que la persuasión es beneficiosa tanto para el emisor como para el receptor de la información.

4 Muñoz Alonso et al. (1990); Capellán de Miguel (2003); Childs (1965), y Monzón Arribas (1987).

5 Chomsky (2002); Cutlip (1994); Herman y Chomsky (1988); Messinger (2012), y Moscovici (1985).

6 Rospir (2010). Las primeras encuestas de opinión fueron elaboradas por el grupo Gallup a finales del periodo de entreguerras.

7 Cabe señalar Collins (2013); Gary (1999); Migliucci (2016), y Sproule (1997). Otros trabajos han analizado los debates que tuvieron lugar en el mismo periodo en otras naciones democráticas, véase por ejemplo García Fernández (2016), y Rodríguez Infiesta (2016). 
es que hasta la fecha ha sido más bien escasa la atención que los estudiosos han dedicado a las diferentes definiciones que se asociaban a principios del periodo de entreguerras a estas nociones, y eso pese a que se trata de unos conceptos que acabarían siendo fundamentales en el contexto de la célebre querella intelectual sobre el control de la opinión de las mayorías democráticas que se desarrolló en los años veinte y treinta ${ }^{8}$.

La aportación del presente artículo será de triple índole. Por una parte, se llevará a cabo un estudio conjunto de las atribuciones que se les asignaba entonces a los conceptos "propaganda" y «opinión pública», cuando en la literatura académica reciente se ha optado generalmente por análisis individualizados o por separado. Por otra parte, la investigación se centrará en un marco cronológico concreto - el periodo de transición entre el estado de guerra y la nueva época de paz (1918-1922) — que hasta el momento ha sido poco abordado por los especialistas en opinión pública y propaganda. Finalmente, se contrastará esta primera labor intelectual con las principales temáticas que acabarían siendo tratadas en el debate erudito sobre persuasión de las masas del periodo de entreguerras.

El periodo histórico analizado abarca desde el fin de la Primera Guerra Mundial (finales de 1918) a la publicación del libro de Walter Lippmann, Public Opinion (1922). La elección de la primera fecha no puede resultar sorprendente, ya que, durante el desarrollo del conflicto, la gran mayoría de los políticos de los principales partidos había apoyado el esfuerzo bélico nacional, participando muchos miembros del mundo de la cultura en las actividades de propaganda que las instituciones federales habían organizado con el fin de reforzar la fidelidad a las políticas gubernamentales. Fue solo tras el fin de las hostilidades cuando diversos políticos e intelectuales comenzaron a cuestionar abiertamente la legitimidad de la propaganda gubernamental y a esbozar inquietudes en relación con la naturaleza de la opinión pública. En cuanto a la publicación de Public Opinion, la literatura académica producida hasta la fecha parece coincidir en que el debate arrancó precisamente con la publicación, en 1922, del libro de Lippmann, considerándose dicha obra como el punto de partida de las reflexiones eruditas sobre la construcción del consenso democrático que caracterizaron la actividad intelectual del periodo de entreguerras ${ }^{9}$. En efecto, la publicación del libro de Lippmann provocó numerosas reacciones en el mundo de la cultura, siendo la más célebre la del filósofo Dewey, con el que Lippmann entabló un largo intercambio de opiniones

\footnotetext{
8 Aunque sí merece la pena señalar obras como Marks (1957), o el ya citado Rospir (2010).

9 Lippmann (1922).
} 
sobre propaganda, educación de la ciudadanía y rol jugado por la opinión pública en el sistema democrático ${ }^{10}$.

Con todo, el presente trabajo pretende demostrar que, anteriormente a la difusión del libro de Lippmann, ya habían sido muy numerosos los intelectuales que habían expresado opiniones —o incluso llevado a cabo unos primeros análisis - sobre el poder de la propaganda. Se trataba generalmente de investigaciones científicas aisladas, o incluso de pequeñas reflexiones contenidas en artículos de prensa cuyo argumento principal tenía muy poco que ver con la persuasión de las masas. $Y$, sin embargo, fue sobre estas primeras observaciones que se fueron formulando las complejas tesis eruditas que marcaron el debate posterior sobre la persuasión de las masas. No es por tanto de extrañar que algunos de los protagonistas de estas primeras prospecciones - Edward Bernays, Zechariah Chafee, John Dewey, Raymond Dodge, Walter Lippmann, etc.- acabaran siendo activos participantes en el debate que se desarrolló durante las dos décadas siguientes.

Con el fin de reconstruir las estructuras semánticas que caracterizaron los primeros años de paz después de la Gran Guerra, se analizarán fuentes documentales que fueron producidas en aquella época por las tres categorías profesionales que más contribuyen (ahora como entonces) a la conformación de la opinión de las masas: los científicos sociales, los periodistas y los políticos. Por una parte, se analizarán los artículos académicos que se publicaron en aquellos años en diferentes revistas científicas norteamericanas, y cuyo objeto de estudio estaba relacionado con el análisis de la propaganda y de la opinión pública. Por aquel entonces dichas cuestiones no se encontraban en el centro del debate científico (todavía no existían instituciones como el Institute for Propaganda Analysis o revistas especializadas como el Public Opinion Quarterly); sin embargo, algunos académicos — expertos en campos como psicología, sociología, historia, economía, estudios literarios, religiosos o militares - ya producían trabajos pioneros que servirían de base para la futura investigación sobre persuasión de las masas ${ }^{11}$. Por otra parte, también se examinará el contenido de los artículos de opinión y de los editoriales que aparecían en destacados periódicos de la época (en particular el New Republic — semanal próximo a los ambientes más progresistas del país-, y el New York Times —un diario con una orientación ideológica

10 Tal y como señala Jansen (2009), los dos autores intercambiaron opiniones, aunque nunca tuvieron posturas antagónicas.

11 Tanto el Institute for Propaganda Analysis como el Public Opinion Quarterly comenzaron sus actividades en 1937. 
más moderada- $)^{12}$. Finalmente, se analizarán las posturas defendidas entonces por algunos de los más notorios intérpretes de la vida política de aquel periodo, comenzando con los presidentes Woodrow Wilson y Warren G. Harding.

Al contrastar las fuentes hemerográficas con la producción científica y los relatos de los dirigentes nacionales, será posible remontarse a las diferentes lógicas discursivas que se generaron entonces alrededor de la delicada temática del control de la información dentro de los sistemas democráticos, lo que vino a constituir el caldo de cultivo del célebre debate sobre propaganda gubernamental del periodo de entreguerras. Lo cierto es que fueron los procesos político-sociales que se desarrollaron durante el conflicto y en los primeros ańos de la posguerra - experiencia propagandística durante la confrontación militar, campańas divulgativas de movimientos políticos subversivos, potencial de los nuevos medios de comunicación etc.- - los elementos fundamentales que contribuyeron a forjar el significado de estos conceptos, formándose así en dicha coyuntura histórica las diferentes posturas sobre los efectos de la propaganda en la opinión pública que constituirían de ahí a pocos años los pilares del debate sobre la creación del consenso en las naciones democráticas.

\section{EL CRECIENTE INTERÉS POR LA «PROPAGANDA» Y LA «OPINIÓN PÚBLICA»}

En los primeros años tras el fin de la Gran Guerra nociones como «propaganda" $\mathrm{y}$ "opinión pública» adquirieron una importancia cada vez más destacada en Estados Unidos, encontrando amplio espacio en periódicos y revistas académicas, y siendo frecuentemente esgrimidas en sus discursos por numerosos dirigentes políticos del país. La creciente atención que estas cuestiones recibieron en dicho periodo histórico estuvo estrechamente relacionada con algunos eventos destacados que tuvieron lugar en aquellos años.

En primer lugar, cabe señalar las diferentes actividades de propaganda que numerosas naciones beligerantes pusieron en marcha durante la Gran Guerra, con las cuales se pretendió influir tanto sobre la opinión pública interna - reforzando los sentimientos nacionalistas - como sobre las naciones enemigas, debilitando la moral de civiles y militares.

12 Desde 1900 a la época objeto de estudio el New York Times respaldó a tres candidatos presidenciales demócratas (Alton Parker, Woodrow Wilson y James Cox) y a dos republicanos (William McKinley y William Taft). 
Muchos autores no pudieron ocultar su preocupación por el papel que había jugado la propaganda durante los años de la guerra en Europa. El politólogo Charles Merriam, por ejemplo, recordaba que había sido la propaganda — «una de las armas más mortales desarrolladas durante la Gran Guerra»la causa principal del desmoronamiento de las tropas italianas en Caporetto (1917). Por su parte, el académico y militar Edward L. Munson, alto mando de la Morale Section del ejército norteamericano, le atribuía a la propaganda la responsabilidad de la caída del imperio zarista $(1917)^{13}$.

Dentro del mundo institucional también se comenzaba a percibir como una gran amenaza el hecho de que unos propagandistas a sueldo de entidades hostiles al sistema democrático norteamericano - como los imperios centrales o el movimiento bolchevique- hubiesen tenido tanto éxito a la hora de manipular la opinión pública de diferentes países. En sus discursos, el presidente Wilson alertó repetidas veces sobre los peligros de la propaganda extranjera. En particular, las campañas de divulgación de los alemanes se convirtieron en la excusa con la que el inquilino de la Casa Blanca intentaba justificar las resistencias que estaba encontrando entre la opinión pública el proyecto de la Sociedad de las Naciones: "It is to the immediate interest of Germany to separate us from our associates in the war, and I know that the opposition to the treaty is most acceptable in those quarters of the country where pro-German sentiment was strongest. I know that all over the country German propaganda has lifted its hideous head again, and I hear the hiss of it on every side» ${ }^{14}$.

Con todo, durante la Primera Guerra Mundial el pueblo norteamericano también fue testigo de la creación de un aparato gubernamental de propaganda, el primero de la historia de Estados Unidos. El Comité de Información Pública, creado por el presidente Woodrow Wilson tras la entrada de Estados Unidos en el conflicto, bombardeó a la población con mensajes patrióticos - trasmitidos a través de pósteres, canciones nacionalistas, artículos de periódicos, etc.- que pretendían invalidar los argumentos de quienes dudaban de que la intervención militar estadounidense en la guerra europea fuese legítima o beneficiosa ${ }^{15}$. Al finalizarse el conflicto fueron muchos los intelectuales que reflexionaron sobre esta primera experiencia de propaganda institucionalizada. Algunos exmiembros del Comité acabaron denunciando que en realidad lo que se había llevado a cabo durante la guerra

Merriam (1919): 541-542, y Munson (1921): 25-26.

14 Discurso en el Hotel Alexandria, Los Ángeles (Estados Unidos), 20-9-1919.

15 Sobre la Primera Guerra Mundial, la opinión pública norteamericana y el Committee on Public Information, véase Kennedy (1980); Ross (1996), y Vaughn (1980). 
había sido una intolerable tarea de manipulación de la opinión pública interna, una iniciativa que parecía del todo incompatible con el sistema democrático norteamericano. El mismo Lippmann, que durante la guerra había sido alistado por el equipo del Comité, aseguró que su actividad también se había caracterizado por maniobras a su juicio inmorales, desde la censura a la compra de periodistas.

During this war the deliberate manufacture of opinion both for export and for home consumption has reached the proportion of a major industrial operation [...] some day the technic must be investigated if the judgments of peoples are to escape persistent exploitation. When the story is told, it will cover a range of subjects extending from legal censorship to reptile press, from willful fabrication to the purchase of writers, from outright subsidy to award of ribbons $s^{16}$.

Pero, sobre todo, en aquellos años comenzó a difundirse el temor de que el Comité de Información Pública podría haberse convertido en un peligroso antecedente a imitar, una inspiración (y tentación) para los dirigentes nacionales. Una cosa era la propaganda en tiempo de guerra, considerada necesaria para la seguridad nacional pese a todos los interrogantes éticos, pero, ¿qué pasaría en el caso de que alguien decidiera reproducir en tiempo de paz lo aprendido durante el conflicto?

El célebre psicólogo experimental Raymond Dodge fue de los primeros en mostrar una cierta intranquilidad ante este escenario. Aseveró que la Gran Guerra había dejado "legados inquietantes», ya que, gracias al Comité de Información Pública, individuos, asociaciones y gobiernos habían descubierto que la publicidad podía ser utilizada «para moldear la opinión pública y controlar las mayorías democráticas $»^{17}$. Se comenzaba a dar por sentado que la propaganda sería empleada, tarde o temprano, para alcanzar objetivos siniestros. Así lo creía, por ejemplo, el profesor Edward K. Strong Jr., del Carnegie Institute of Technology: «[...] people generally have become conscious of propaganda as a great tool or method for influencing others [...] If propaganda were a means of influencing others along lines only of benefit to society, it could be hailed with great acclaim. But unfortunately it can also be employed for dishonest and socially vicious programs, just as well as for honest and worthwhile movements» ${ }^{18}$.

16 "The Political Scene», The New Republic, 22-3-1919, p. 7.

17 Dodge (1920): 241.

18 Strong (1922): 234-235. 
Otro factor significativo a la hora de comprender el creciente interés por la cuestión de la persuasión de la opinión pública fue la aparición de nuevas tecnologías que, precisamente en aquellos años, estaban revolucionando el mundo de los medios de comunicación. Antenas y telégrafos — afirmaba en 1919 el presidente Wilson- se habían convertido en "los símbolos de la época ${ }^{19}$. La radio comenzaba a dar sus primeros pasos, estrenándose en Detroit y Pittsburgh en 1920 las primeras estaciones comerciales. El cine, por aquel entonces, ya había demostrado todo su potencial como herramienta de persuasión, habiendo utilizado el Comité de Información Pública películas de propagada durante la Gran Guerra. Fue aquella también la época en la que la publicidad se reafirmó como el motor de un sistema económico que en Estados Unidos se sustentaba cada vez más en el consumo de masas. No es de extrañar que fuera en este mismo periodo cuando apareció la nueva profesión de experto en relaciones públicas, cuyo miembro más célebre fue sin duda Edward Bernays. ${ }^{20}$

La aparición de estos nuevos medios ofrecía oportunidades interesantes a la hora de mejorar la transmisión de información desde las instituciones a la ciudadanía, pero también provocó recelo, pues se temía que las nuevas tecnologías de la comunicación acabasen siendo utilizadas por organizaciones antidemocráticas con el fin de manipular la opinión pública.

Un tercer factor a tener en cuenta, en efecto, fue el llamado red scare. Entre 1917 y 1920, en la estela de la Revolución bolchevique en Rusia, diferentes movimientos de la izquierda radical promovieron un gran número de manifestaciones tanto en Europa como en Estados Unidos. Huelgas y motines se sucedían con preocupante periodicidad, provocando gran aprensión en determinados sectores de la sociedad norteamericana.

En la prensa se multiplicaron las noticias relacionadas con las actividades de divulgación que algunos movimientos radicales estaban llevando a cabo en el país. La tentación, para muchos periodistas y políticos, fue la de negar el malestar social y económico que vivían las capas más desfavorecidas de la población, atribuyendo los frecuentes disturbios exclusivamente a un intento de derrocamiento de las instituciones democráticas por parte de agentes externos.

El New York Times era uno de los periódicos que más convencido se mostraba de que la principal causa de los motines era la labor de propaganda de los bolcheviques. La búsqueda del término "propaganda» en el archivo online del New York Times proporciona más de 5700 resultados para el periodo comprendido entre noviembre de 1918 y diciembre de 1922, apareciendo dicha palabra en más de 350 editoriales y 800 artículos de portada. Un gran número

19 Discurso en el Coliseum de Des Moines (Estados Unidos), 6-9-1919.

20 Davison (2016). 
de dichos artículos denunciaba las campañas de persuasión a las que estaba sometido el pueblo estadounidense. ${ }^{21}$

Este análisis, obviamente, era compartido por el presidente Wilson. Desde su punto de vista, resultaba evidente que al Gobierno no se le podía atribuir ninguna responsabilidad por las protestas, pues su equipo en absoluto había descuidado las necesidades de los estratos sociales económicamente más vulnerables. En realidad, el indiscutible desencadenante de los disturbios era la propaganda procedente de Rusia.

That poison is running through the veins of the world, and we have made the methods of communication throughout the world such that all the veins of the world are open and the poison can circulate. The wireless throws it out upon the air. The cable whispers it underneath the sea. Men talk about it in little groups, men talk about it openly in great groups not only in Europe but here also in the United States. There are apostles of Lenin in our own midst. I can not imagine what it means to be an apostle of Lenin. It means to be an apostle of the night, of chaos, of disorder ${ }^{22}$.

\section{OPINIÓN PÚBLICA Y PROPAGANDA: INTENTOS DE CONCEPTUALIZACIÓN}

Tras el fin de la Primera Guerra Mundial, el significado de «opinión pública» no estaba todavía rotundamente definido. Los sociólogos norteamericanos Charles H. Cooley y Charles A. Ellwood fueron de los primeros en aventurar unas definiciones. El primero hablaba de un proceso orgánico de interacción, comprensión e influencia mutua entre los diferentes miembros de un grupo; el segundo, de una coordinación hacia una determinada dirección de juicios individuales separados, que finalmente llevarían a un juicio colectivo $^{23}$. El psicólogo social William McDougall postulaba la existencia de una «mente grupal», la cual hacía que las personas actuasen en público de forma diferente a como lo hacían en privado ${ }^{24}$.

21 Véase, por ejemplo, «Bolshevist Propaganda», The New York Times, 23-1-1919, p. 12; "New York Bolsheviki», The New York Times, 27-5-1919, p. 14; «Race Riots», The New York Times, 28-7-1919, p. 10; "The Impunity of Bolshevism», The New York Times, 10-9-1919, p. 12, y «The Reds», The New York Times, 11-9-1919, p. 12.

22 Discurso en el Fairgrounds Auditorium de Billings (Estados Unidos), 11-9-1919.

23 Cooley (1918): 378-379, y Ellwood (1920): 74-75.

24 McDougall (1920). 
Lo que más llama la atención es que en aquel periodo histórico el significado de «opinión pública» no parecía asociarse con un conjunto de actitudes y percepciones, sino con el poder de las normas sociales y de la ética comunitaria. En varias obras publicadas durante los años de la Primera Guerra Mundial y a comienzo del periodo de entreguerras, el sociólogo E. Robert Park definió la opinión pública como una «fuente de control social»" ${ }^{25}$. De la misma forma, para el coronel Munson se trataba de una «poderosa agencia para el control de la conducta ${ }^{26}$. El general de brigada de las fuerzas armadas estadounidenses, Andrews Lincoln, aseguraba que, en circunstancias normales, los individuos muestran «deferencia» hacia la opinión pública: «Obedience to law is normally the result of public opinion. When this fails in individual cases, the police power is employed. Where many men join together in open violation of law, in defiance of public opinion, they are no longer quite reasonable, and their misconduct may soon pass the power of the civil authorities to control. To meet this contingency every government maintains a military force ${ }^{27}$.

En cuanto al concepto de propaganda, a comienzos del periodo de entreguerras este término se consideraba — esas eran las palabras empleadas por el New York Times - «perverso» y «difícil de definir»". En un informe sobre la labor de los propagandistas elaborado en 1919 por los expertos del aparato militar estadounidense, se asociaba a la noción de "propaganda» toda una serie de heterogéneas actividades como la difusión de falsas alarmas, las insinuaciones calumniosas y la propagación de información cuyo fin era la distorsión, exageración o supresión de la verdad.

All of these are engaged in activities to which the word "propaganda" has not been applied; yet they illustrate it perfectly. There has been no question of legal authority for the punishment of these acts. Propaganda has therefore a legal status, though under other names. If propaganda against an individual's reputation, the safety of a crowd of people in a theatre, the prosperity of a local bank, is dangerous to the public welfare, by so much the more is it dangerous when it attacks the honor of a nation, the lives of its soldiers, the fate of its armies and the liberty of the whole people ${ }^{29}$.

\footnotetext{
Park (1915); Park (1923), y Park y Burgess (1921).

Munson (1921): 162.

Lincoln (1920): 175-176.

28 "The Books of Our Enemies», The New York Times, 1-1-1920, p. 14.

29 United States War Dept. General Staff (1919): 1.
} 
Algunos ilustres exponentes del mundo académico de la época intentaron alcanzar unas definiciones algo más concretas. El psicólogo Raymond Dodge, por ejemplo, hablaba del «arte de construir la mente del otro, de ganar adherentes a unos principios, de obtener apoyos para una opinión o un curso de acción ${ }^{30}$.

Algunos autores establecieron un lazo directo entre el sistema de la democracia, por una parte, y de la difusión de las actividades de propaganda, por otra. El filósofo Everett Martin, por ejemplo, subrayó que la libertad de hacer propaganda era, junto con otras libertades como el derecho al voto o el libre comercio, una de las características intrínseca del régimen democrático ${ }^{31}$.

Pero, por lo general, resultaba evidente que para muchos autores de la época la noción «propaganda» había adquirido una connotación negativa. El mismo director del Comité de Información Pública, George Creel, aseguraba que esta palabra era sinónimo de términos como «engaño y corrupción». Por supuesto, en su opinión estas expresiones representaban adecuadamente las actividades de manipulación llevadas a cabo durante el conflicto mundial por los alemanes, pero en absoluto podían utilizarse para describir la tarea que su Comité había desempeńado durante la misma guerra. Creel aseguraba que en este caso no se había tratado de propaganda, sino de una labor «informativa y educacional $\aleph^{32}$.

Desde fechas muy tempranas, los dos conceptos — propaganda y opinión pública- comenzaron a ser analizados de forma conjunta. Las múltiples experiencias propagandísticas de la guerra y la irrupción de las nuevas tecnologías de la información en el mundo moderno inevitablemente hacían nacer nuevos interrogantes sobre la capacidad de la opinión pública para resistirse ante las campañas de los nuevos medios de comunicación.

El punto de partida de los análisis sobre los efectos de la propaganda en la opinión pública fueron algunas obras que, desde hacía ya algunos años, se preocupaban de examinar la forma en la que el ser humano reacciona ante los impulsos exteriores. ¿Cómo se llega a la toma de decisión? ¿La conducta de un individuo es consecuencia de su capacidad de analizar lógicamente el entorno o es por el contrario determinada por su esfera más pasional?

Tras el fin de la guerra, por ejemplo, se publicó The Science of Power, libro póstumo del sociólogo Benjamin Kidd, en el que se afirmaba que «la civilización no descansa en la razón sino en la emoción» ${ }^{33}$. Unos pocos ańos antes, el

\footnotetext{
Dodge (1920): 242.

Martin (1920): 261

Creel (1920): 1 .

Kidd (1919): 117.
} 
neurocirujano Wilfred Trotter había llegado a teorizar que en los hombres y mujeres de la época contemporánea todavía era muy fuerte el primitivo «instinto de la manada ${ }^{34}$.

Partiendo de estas premisas, muchos científicos plantearon fuertes dudas sobre la capacidad del pueblo para gestionar los asuntos de la res publica. Edward Strong, profesor de psicología aplicada en la Universidad de Stanford, recordaba que el ciudadano medio confesaba saber muy poco sobre asuntos nacionales e internacionales ${ }^{35}$. El filósofo Everett Martin iba incluso más allá, sugiriendo que los propagandistas podían construir, campańa tras campaña, la forma de pensar de la ciudadanía.

Public opinion is manufactured just as brick are made. Possibly a slightly better knowledge of mechanical engineering is required for making public opinion, but the process is the same. Both can be stamped out in the quantity required, and delivered anywhere to order. Our thinking on most important subjects today is as little original as the mental processes of the men who write and the machines which print the pages we read and repeat as our own opinions ${ }^{36}$.

Sin embargo, otros expertos defendían posturas radicalmente diferentes, asegurando que la opinión pública en realidad jugaba un rol activo en la composición de las reglas comunitarias. De acuerdo con Ellwood, en las sociedades civilizadas - y en particular en las democracias - la opinión pública se encontraba detrás de las instituciones, las leyes y las costumbres, unos «hábitos sociales sancionados por la opinión de grupo »37. Muchos autores defendían la capacidad de la ciudadanía para juzgar los asuntos de Estado con discernimiento y mesura, destacándose el lugar central que la opinión pública tenía en la elaboración de políticas tanto a nivel nacional como internacional. John Dewey, por ejemplo, expresaba «confianza en la sabiduría y buena voluntad de los pueblos $\aleph^{38}$.

En determinadas ocasiones la opinión pública venía descrita como el actor esencial del sistema democrático, una fuerza capaz de determinar y dirigir el comportamiento de los políticos. El New York Times aconsejaba a los candidatos a la Casa Blanca «escuchar la voz del hombre de la calle», ya que

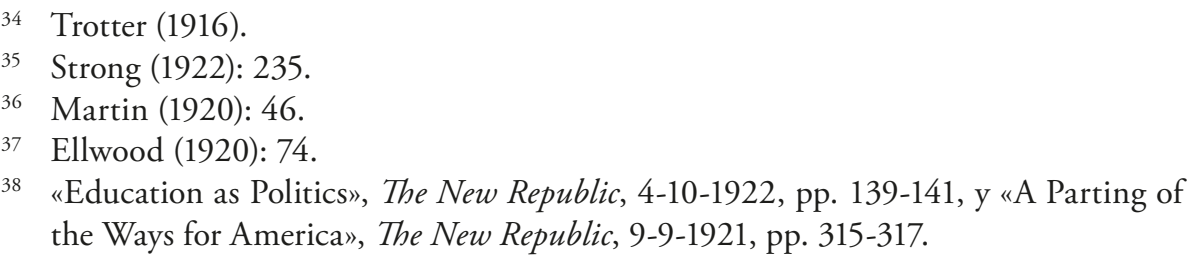


finalmente eran los ciudadanos quienes definían la agenda política de las campańas presidenciales ${ }^{39}$. En un artículo publicado por el periódico neoyorquino, Bernays refería que en la Conferencia de Paz de París (1919) los delegados de pueblos como los árabes o los lituanos no hablaban de sus reivindicaciones de autodeterminación con los diplomáticos de Wilson, sino que se dirigían directamente a la prensa norteamericana, pues sabían que el presidente respaldaría sus aspiraciones solo en el caso de que estas contasen con la bendición de la opinión pública estadounidense ${ }^{40}$.

\section{LA CREACIÓN DEL CONSENSO: UNA NUEVA HERRAMIENTA PARA LOS DIRIGENTES POLÍTICOS}

En un régimen político como el norteamericano — considerado como uno de los grandes pilares de las democracias modernas - se daba por sentado que el poder de los dirigentes políticos emanaba de la opinión pública. Los inquilinos de la Casa Blanca solían ensalzar en sus discursos el poder de la opinión pública, unas alabanzas que - considerando que habían sido los ciudadanos quienes los habían elegido para el gobierno de la nación — sonaban en realidad como unos evidentes intentos de autolegitimización. Aceptando la nominación republicana para la carrera hacia la Casa Blanca, el futuro presidente Harding aclaró que, gracias a la Constitución norteamericana, en Estados Unidos se garantizaba el gobierno de la opinión pública: «Under the Constitution we have the charted way to security and perpetuity. We know it gave to us the safe path to a developing eminence which no people in the world ever rivalled. It has guaranteed the rule of intelligent, deliberate public opinion expressed through parties. Under this plan, a masterful leadership becomingly may manifest its influence, but a people's will still remains the supreme authority» ${ }^{41}$.

De la misma forma, Wilson se definía como un mero «portavoz, un instrumento» que intentaba «absorber las influencias de la opinión pública en los Estados Unidos» ${ }^{42}$. Wilson comparaba constantemente la opinión pública con «un tribunal» que ejercía «una fuerza destructiva hacia las causas injustas» ${ }^{43}$.

39 «Who Makes the Issue?», The New York Times, 21-12-1919, p. 33.

40 «From a Peace Conference Reporter's Notebook», The New York Times, 6-4-1919, p. 77.

41 Discurso de aceptación de la nominación republicana, 12-6-1920.

42 Discurso en el Auditorio Municipal de Denver (Estados Unidos), 25-9-1919.

43 Discurso en el Estadio de Balboa Park, San Diego (Estados Unidos), 19-9-1919. 
Aseguraba que la Sociedad de las Naciones — cuyo establecimiento se convirtió en su máxima prioridad política de la posguerra - sería el «tribunal de la opinión pública de la humanidad». A esta opinión pública global le atribuía poderes casi divinos, ya que finalmente esta ejercería una "presión terrible e irresistible» sobre los gobiernos, una «influencia purificadora y redentora» sobre la sociedad, lo que les permitiría «corregir los errores del pasado» tanto a los ciudadanos como a los líderes políticos ${ }^{44}$.

Esta postura, sin embargo, era desafiada por una parte importante del mundo de la cultura. Eran numerosos los intelectuales que reivindicaban para sí mismos el papel de portavoces de la opinión pública. El académico y diplomático David Hill se oponía a la idea de que el presidente de Estados Unidos representase «la voluntad preponderante de la nación».

It will perhaps be said that, when the President has confidence that he is acting in the public interest and believes he will be sustained by the popular will, he ought not to be under any restraint [...] The major premise in this argument is a bold appeal for autocratic, as distinguished from representative, government. It may be predicated of an Emperor with as much force as of a President. It is a false doctrine, accompanied by a retinue of false assumptions ${ }^{45}$.

Ellwood, por su parte, defendía que la opinión pública se correspondía con el «madurado juicio de los dirigentes y especialistas que están en estrecho contacto con el público», dejando así entender que el punto de vista de los expertos, no solo el de los políticos, estaba en simbiosis con la voluntad popu$\operatorname{lar}^{46}$. En un editorial del New York Times se afirmaba que la voluntad de la opinión pública se reflejaba perfectamente en la opinión mayoritaria expresada por los periódicos con mayor difusión ${ }^{47}$.

Los políticos, de todos modos, no se conformaban con ser los representantes de la opinión pública. De acuerdo con las denuncias de numerosos miembros del mundo de la cultura de la época, su verdadero objetivo era influirla y conformarla. Lo cierto es que durante la guerra los órganos institucionales, tanto los federales como los locales, habían construido un robusto aparato

44 Discurso desde la "plataforma trasera», Sacramento (Estados Unidos), 22-9-1919; discurso en el Palace Hotel, San Francisco (Estados Unidos), 18-9-1919, y discurso en el Mormon Tabernacle, Salt Lake City (Estados Unidos), 23-9-1919.

45 Hill (1919): 18-28.

46 Ellwood (1920): 75.

47 «Public Opinion on Government Ownership», The New York Times, 2-6-1919, p. 14. 
administrativo — formado esencialmente por agentes de prensa — dedicado a la transmisión de información al público estadounidense, un fenómeno que no se detuvo con el fin de las hostilidades ${ }^{48}$.

A principios del periodo de entreguerras varios autores comenzaron a mostrar inquietud por el imparable crecimiento de las estructuras de propaganda en el seno de las instituciones federales. En la revista Religious Education se aseveraba que «el extraordinario desarrollo de los medios de comunicación a través de la prensa, los correos y las películas, la facilidad del transporte y la concentración de dinero» habían otorgado «un poder sin precedentes» a quienes controlaban "las vías del pensamiento público ${ }^{49}$. El periodista George Brown recordaba que, durante el periodo de la guerra, «cada pequeño funcionario tenía un agente de prensa propio, para servir a sus fines egoístas y aumentar su propio prestigio», mientras que en la American Economic Review se leía que el trabajo de propaganda del Gobierno había alcanzado "proporciones tremendas y abrumadoras" ${ }^{50}$.

Se denunciaba asimismo que los agentes de prensa de los varios departamentos lograban alterar los contenidos de los artículos de periódicos y revistas. En el ideario norteamericano la prensa libre se había entendido tradicionalmente como un implacable vigilante del poder al servicio del pueblo, además de una dispensadora de información y conocimiento para la ciudadanía. Y, sin embargo, cada vez más se difundía entonces la sensación de que este pilar del sistema democrático estaba siendo corrompido por los dirigentes políticos. Roscoe Brown —un experto en el mundo del periodismoaseguraba que para periódicos y revistas la propaganda era un "parásito», siendo el agente de prensa «su instrumento de infección " ${ }^{51}$.

El mundo intelectual se mostraba además altamente escéptico en cuanto a la capacidad de los ciudadanos de defenderse de la labor divulgativa de las autoridades federales. En algunos artículos de prensa se destacaba que el ciudadano de a pie no hacía ningún ejercicio de reflexión crítica ante las campañas informativas del Gobierno ${ }^{52}$. El periodista Frederick Schrader aseguraba que la propaganda se estaba imponiendo sobre la «ignorancia, indiferencia y credulidad del pueblo norteamericano $»^{53}$.

\footnotetext{
48 Canes-Wrone (2006): 13.

49 Religious Education (1919): 408.

50 Brown (1919): 801, y Boyle y Smith (1919): 56.

51 Brown (1921): 610.

52 "Europe's Misery and America's Complacency», The New Republic, 12-9-1919, pp. 305-307, y «Some Recent Experience», The New Republic, 12-9-1919, pp. 318-319.

53 Schrader (1920): 9.
} 
Pero ¿qué tipo de actividades divulgativas se organizaban desde el Gobierno? En estos primeros años de paz fueron muchas las protestas - por parte de periodistas y otros intelectuales - por las campañas que las instituciones federales estaban supuestamente llevando a cabo. Muchos pedagogos, por ejemplo, alertaron sobre la propaganda que el Gobierno estaba realizando en los centros educativos. El National Industrial Conference Board protestó por el material propagandístico que, bajo petición de Wilson, había sido distribuido durante el último año de guerra en las escuelas primarias, intermedias y superiores, lo que fue definido como «un abuso de la función de publicidad gubernamental $\aleph^{54}$.

El New Republic, por su parte, denunciaba la existencia de campañas de denigración contra los trabajadores y sus representantes.

During and since the war they polluted the sources of public opinion by censorship and propaganda. The censorship has passed, but propaganda remains. It persists in a particularly virulent form in our own country [...]. The government, with the support for the most part of the politicians, the newspapers and the majority of well-to-do and educated people, is seeking by mass compulsion to terrorize public opinion, to confuse radical thought with treason, and to identify intellectual obscurantism and timidity with loyal Americanism ${ }^{55}$.

No puede además olvidarse que durante la guerra fueron aprobados el Espionage Act y el Sedition Act, que limitaron drásticamente la libertad de expresión en tiempo de guerra. El primero pretendía castigar toda tentativa de provocar insubordinación entre los militares, así como cualquier tipo de respaldo a la causa de los enemigos del país. Con el segundo se perseguían los ataques verbales contra el Gobierno, la bandera o las fuerzas armadas ${ }^{56}$.

En 1919 Wilson propuso lanzar una operación de publicidad — financiada con dinero público - para convencer al pueblo de la necesidad de extender en tiempo de paz el Food Control Act de $1917^{57}$.

A comienzo del periodo de entreguerras, en suma, se fue creando, dentro del mundo de la cultura estadounidense, un fuerte clima de resistencia con respecto a las iniciativas divulgativas del Gobierno. Muy significativo a este propósito es un artículo que el New York Times publicó en noviembre de 1918, en el cual se expresaba irritación por la decisión del Comité de Información

\footnotetext{
National Industrial Conference Board (1919): 3.

"Freedom of Opinion and the Clergy", The New Republic, 11-2-1920, pp. 303-305.

Manz (2007).

57 Séptimo mensaje anual al Senado y la Cámara de los Representantes, 2-12-1919.
} 
Pública de definir una de sus campañas en el extranjero como una official press mission.

We have heard of an official press in Austria and Germany. Is there an official press in the United States? If there is, by what authority, founded upon what constitutional power of the Government, does it rest? Its ultra-marine purpose is described as "to interpret the work of the Peace Conference by keeping up world-wide propaganda to disseminate American accomplishments and American ideals". Is an official press, a Government press, among those ideals and accomplishments? ${ }^{38}$.

Pero no todos los científicos rechazaban las actividades informativas del Gobierno. Para algunos, en efecto, las nuevas tecnologías proporcionaban unas posibilidades pedagógicas que tenían que ser aprovechadas por las instituciones. Harold Larrabee, por ejemplo, se decía favorable a la creación de una labor informativa que permitiese propagar estándares morales y valores religiosos entre los ciudadanos. Una buena muestra de ello sería la difusión de películas de propaganda, convirtiendo así el cine comercial en una herramienta educativa. Con el fin de alcanzar una amplia difusión de dichos contenidos, el filósofo sugería recurrir incluso a la manipulación de los espectadores: «To introduce propaganda films of any sort into the commercial theater it will be necessary either (a) to pay for their exhibition; (b) to camouflage their real nature; (c) to make them sensationally popular in appeal; (d) to conduct an advertising campaign creating a demand; or (e) to control the theaters in which they are to be shown $"$.

Una especial mención la merecen las reacciones del mundo intelectual de la época ante un escenario que de ahí a unos pocos años se haría muy popular: la posibilidad de que el Gobierno federal organizase una labor de contrapropaganda para contrarrestar las campañas de los movimientos subversivos (así se haría desde finales de los años treinta para neutralizar la difusión en Estados Unidos de la doctrina nazi).

En el New Republic, el periodista Frank Irving Cobb se declaraba contrario a la idea de que al Gobierno le incumbiese defender al pueblo de la propaganda antidemocrática, tachando esta actividad de paternalismo autoritario ${ }^{60}$. Con todo, en la misma revista también se admitía que al fin y al cabo era muy sutil la línea que separaba la libertad de expresión, por una parte, y las crimi-

58 "An American “Official Press"”, The New York Times, 22-9-1918, p. 12.

59 Larrabee (1920): 154.

60 «The Press and Public Opinion», The New York Times, 31-12-1919, pp. 144-147. 
nales campañas de propaganda destinadas al derrocamiento de las instituciones, por otra ${ }^{61}$.

Se trataba en realidad de un asunto extremadamente controvertido. ¿Hasta qué punto era legítimo para el Gobierno federal proteger a la opinión pública de quienes pretendían menoscabar sus libertades? Y, sobre todo, ¿a quién le correspondía marcar el límite entre la libertad de expresión —que el Estado tenía que amparar-y la propaganda subversiva, que por el contrario tenía que ser reprimida? Ya a caballo entre los años diez y veinte, el académico Zechariah Chafee publicó importantes estudios científicos sobre esta cuestión ${ }^{62}$. Consideraba extremadamente importante que se establecieran «los verdaderos límites de la libertad de expresión", para que los ciudadanos conociesen lo que se podía decir, y los gobiernos supiesen lo que se podía censurar. Dichos límites tenían que ser delimitados a partir de un atento examen de la Constitución norteamericana, una labor que por supuesto tenía que ser confiada a los especialistas en jurisprudencia. Por ejemplo, Chafee consideraba que durante la Gran Guerra los políticos habían limitado la libertad de expresión de forma razonable y justificada, y que hubo sin embargo evidentes excesos, a la hora de ejecutar las leyes, por parte de las autoridades gubernamentales.

[...] action in proportion to the emergency was justified, but we have censored and punished speech which was very far from direct and dangerous interference with the conduct of the war. The chief responsibility for this must rest, not upon Congress which was content for a long period with the moderate language of the Espionage Act of 1917, but upon the officials of the Department of Justice and the Post-office, who turned that statute into a drag-net for pacifists, and upon the judges who upheld and approved this distortion of law ${ }^{63}$.

Esta línea de argumentación ofrecía interesantes posibilidades a los defensores de la propaganda defensiva. Con el tiempo se iba delineando la idea de que el Estado democrático sí tenía las prerrogativas constitucionales para intervenir en campos tan delicados como la limitación de los derechos individuales o la propagación, entre la opinión pública interna, de información, opiniones y doctrinas.

Por ejemplo, ya a partir de finales de los años diez fueron numerosos los intelectuales que se mostraron favorables a la posibilidad de luchar en contra de la propaganda antiestadounidense que se desarrollaba en otras naciones

61 "What Is Left of Free Speech», The New Republic, 10-12-1919, pp. 50-52.

62 Chafee (1919), y Chafee (1920).

63 Chafee (1919): 933. 
— por ejemplo, en Iberoamérica — con una adecuada contrapropaganda ${ }^{64}$. Se comenzaba a percibir que incluso un Estado democrático — por su misma naturaleza reticente a la implementación de medidas consagradas al adoctrinamiento de la población — podía verse obligado — a su pesar y por ineludibles exigencias defensivas - a una forzosa guerra preventiva de propaganda.

¿Podía además un Gobierno responsable desechar un instrumento que, durante los años de la guerra, se había revelado tremendamente eficaz para la consecución de vitales intereses nacionales? En varios artículos de prensa, en efecto, se declaraba entusiásticamente (y quizá exageradamente) que durante el conflicto la labor del Comité de Información Pública había sido tan importante para la victoria final como las mismas maniobras bélicas. Si en un editorial de New Republic se recordaba que durante el conflicto una «audaz contrapropaganda» había permitido poner fin a la actitud derrotista de las naciones aliadas, en un artículo de opinión publicado por la misma revista se atribuía a los intelectuales del Comité de Creel el mérito del desplome de Austria y Alemania ${ }^{65}$. Por su parte, Larrabee aseveraba que las películas que el Gobierno produjo durante la guerra podían ser tomadas como ejemplos de propaganda exitosa ${ }^{66}$.

Estas campañas se presentaban a menudo al público como algo sumamente moral, lo que demuestra que todavía en esta época había autores que le otorgaban un matiz positivo al término «propaganda». En el prefacio del libro Adventures in Propaganda se podía leer que, durante la Gran Guerra, por primera vez en la historia de las operaciones militares, la «verdad» había sido utilizada "como arma afectiva»" ${ }^{67}$. El New Republic, por su parte, les proponía a los trabajadores que conquistasen a la opinión pública a través de campańas de persuasión, una estrategia que les permitiría alcanzar las mejoras laborales que necesitaban ${ }^{68}$.

\section{CONCLUSIÓN}

Tras el fin de la Primera Guerra Mundial la temática de la manipulación de la ciudadanía atrajo la atención de numerosos miembros del mundo político

\footnotetext{
64 Perry (1920): 40.

65 "The Let Down», The New Republic, 5-4-1919, pp. 296-297, y «The Psychology of German defeat», The New Republic, 1-2-1919, pp. 23-24.

66 Larrabee (1920): 145.

67 Blankenhorn (1919): V.

68 "Labor Facing the Future», The New Republic, 28-6-1922, pp. 122-123, y «A Gap in Labor Policy", The New Republic, 24-12-1919, pp. 100-102.
} 
y cultural, que reflexionaron sobre el papel y función de la opinión pública o sobre los peligros y oportunidades de la propaganda, adelantando - y propiciando- el célebre debate sobre construcción del consenso democrático que arrancó con la publicación, en 1922, del libro de Lippmann, Public Opinion.

El examen de las fuentes que se produjeron en aquellos primeros años de paz —artículos periodísticos, discursos políticos, obras científicas- nos indica que las primeras reflexiones sobre estas temáticas estaban estrechamente vinculadas con las grandes problemáticas sociopolíticas de aquel periodo, desde las campañas propagandísticas de la guerra a los disturbios provocados por los movimientos radicales, pasando por la consolidación de medios de comunicación particularmente eficaces en la tarea de la persuasión de las masas. Como siempre cuando se analizan las inquietudes que surgen en el seno del mundo intelectual, resulta imposible separarlas de los desafíos, aprensiones y oportunidades propios de las épocas en las que dichos intelectuales vivieron y produjeron sus obras.

En estos primeros años de paz, en suma, se creó un ambiente propicio para el establecimiento de un gran debate sobre propaganda. Lippmann, Dewey, Bernays y los otros protagonistas de la querella sobre la manipulación de la ciudadanía no divisaron unos peligros que hasta entonces habían sido desatendidos. En realidad, su mérito fue el de realizar unas reelaboraciones sofisticadas y sistemáticas a partir de las numerosas reflexiones que diversos intelectuales habían ido planteando en orden disperso durante los primeros años que siguieron al fin del conflicto.

En cuanto a las estructuras semánticas de estas primeras prospecciones, en aquella época se formaron interpretaciones muy divergentes en cuanto al significado de las nociones «opinión pública» y "propaganda», revelándose así toda la complejidad de dichos fenómenos, además de sus contradicciones intrínsecas, algo que también se señalaría en el debate posterior.

En estos primeros ańos de paz se dio un radical giro interpretativo en la valoración de dichos conceptos. La experiencia de la guerra y los peligros de las campañas divulgativas de entidades políticas hostiles llevaron a muchos autores de la época a justificar el establecimiento de campańas de propaganda — entendidas en este caso como actividades pedagógicas - cuyo cometido habría sido el de combatir los engaños perpetrados por los enemigos de la República. Se trataba así de glorificar la labor de la propaganda estadounidense, a la vez que se tachaba de inmoral a la propaganda — esta vez al término se le daba connotación despectiva - de todas aquellas entidades a las que se les podía tachar de antidemocráticas o antiestadounidenses.

En cuanto al concepto de "opinión pública», para algunos autores esta seguía siendo la indiscutible protagonista del sistema democrático. Se daba 
por sentado que la ciudadanía contaba con capacidades y conocimientos suficientes como para interpretar y comprender las grandes problemáticas en las que se veía sumida la nación, delegando el pueblo la administración de la República a los mejores representantes políticos. Y, sin embargo, fue en este periodo cuando comenzó a difundirse una visión más pesimista de la opinión pública, en la que se destacaba la escasa capacidad de resistencia que esta tenía ante la manipulación informativa. En particular, en el mundo académico se incrementó el número de especialistas que la consideraban como una entidad más bien pasiva, fácilmente influenciable por los propagandistas.

También creció en este periodo la preocupación por la posibilidad de que los dirigentes políticos pudiesen aprovecharse del enorme poder de los nuevos medios de comunicación. El mundo intelectual por aquel entonces ya tenía claro que las campańas de propaganda en el exterior eran esenciales para la seguridad del país, así como se aceptó la necesidad de realizar campañas informativas en el interior en caso de grave emergencia nacional. Sin embargo, seguía existiendo mucha controversia ante la idea de que se pudiesen llevar a cabo operaciones de propaganda destinadas a la opinión pública interna en tiempo de paz. Fue precisamente en estos años cuando comenzó a hacerse concreta la posibilidad de que el Gobierno federal acabase estableciendo una estructura permanente de propaganda consagrada a la persuasión de la ciudadanía, un aparato institucional de divulgación informativa que trabajaría tanto durante las crisis bélicas como en tiempo de paz.

¿Sería compatible dicha estructura con el sistema democrático? ¿Tendría la opinión pública la capacidad de asimilar las campañas pedagógicas, rechazando al mismo tiempo las manipulaciones y la demagogia? ¿Sería la contrapropaganda un arma legítima y eficaz para contrarrestar las campañas informativas de los enemigos de la República? ¿Y qué papel deberían desempeñar en dichas estructuras gubernamentales de persuasión los científicos sociales y los periodistas? El debate sobre la creación del consenso de masas estaba a punto de arrancar. Los elementos fundamentales que lo caracterizarían ya habían sido trazados durante estos primeros ańos de paz tras el fin de la Gran Guerra.

\section{Bibliografía}

Bernays, E. (1923). Crystallizing Public Opinion. New York: Liveright.

Black, J. (2001). Semantics and Ethics of Propaganda. Journal of Mass Media Ethics, 16 (2-3), 121-137. Disponible en: https://doi.org/10.1207/S15327728JMME1602\&3_4.

Blankenhorn, H. (1919). Adventures in Propaganda: Letters from an Intelligence Officer in France. Boston: Houghton Mifflin. 
Boyle, J. E., y Smith, G. C. (1919). Effect of Government Control on Marketing Methods and Costs: Discussion. The American Economic Review, 9 (1), 56-60.

Brown, G. R. (1919). The Lynching of Public Opinion. The North American Review, 209 (763), 795-802.

- (1921). The Menace to Journalism. The North American Review, 214 (792), 610-618.

Canes-Wrone, B. (2006). Who Leads Whom?: Presidents, Policy, and the Public. Chicago: University of Chicago Press.

Capellán de Miguel, G. (2003). El giro científico (1870-1910): hacia un nuevo concepto de opinión pública. Historia Contemporánea, 27, 719-732.

Chafee, Z. Jr. (1919). Freedom of Speech in War Time. Harvard Law Review, 32 (8), $932-$ 973. Disponible en: https://doi.org/10.2307/1327107.

- (1920). Freedom of Speech. Nueva York: Harcourt, Brace and Howe.

Childs, H. L. (1965). Public Opinion: Nature, Formation and Role. Princeton: D. Van Nostrand Company.

Chomsky, N. (2002) [1997]. Media Control: The Spectacular Achievements of Propaganda. New York: Seven Stories Press.

Collins, S. (2013). Propaganda Studies, The US Interwar Years. En A.N. Valdivia (ed.). The International Encyclopedia of Media Studies (pp. 578-609). Malden, MA: Wiley Blackwell.

Cooley, C. H. (1918). Social Process. New York: Charles Scribner's Sons.

Creel, G. (1920). How We Advertised America: The First Telling of the Amazing Story of the Committee on Public Information that Carried the Gospel of Americanism to Every Corner of the Globe. New York and London: Harper \& Brothers.

Cunningham, S. B. (2002). The Idea of Propaganda: A Reconstruction. Westport, CT: Praeger/Greenwood.

Cutlip, S. M. (1994). The Unseen Power: Public Relations, A History. Hillsdale, N.J.: Lawrence Erlbaum Associates.

Davison, A. (2016). Shaping the Public Mind: Edward Bernays and the Rise of Public Relations [tesis doctoral]. Georgia Southern University. Disponible en: http://digitalcommons. georgiasouthern.edu/honors-theses/172.

Dewey, J. (1927). The Public and its Problems. New York: Holt.

Dodge, R. (1920). The Psychology of Propaganda. Religious Education, 15 (5), 241-252. Disponible en: https://doi.org/10.1080/0034408200150502.

Ellwood, C. A. (1920). The Formation of Public Opinion. Religious Education, 15 (2), 73-80. Disponible en: https://doi.org/10.1080/0034408200150201.

Gary, B. (1999). The Nervous Liberals: Propaganda Anxieties from World War I to the Cold War. New York: Columbia Univeristy Press.

García Fernández, H. (2016). De Masterman a Orwell: el debate británico sobre la propaganda en democracia, 1914-1945. Rubrica Contemporanea, 5 (10), 25-44.

Herman, E. S. y Chomsky, N. (1988). Manufacturing Consent: The Political Economy of the Mass Media. New York: Pantheon Books.

Hill, D. J. (1919). In the Valley of Decision. The North American Review, 210 (764), 18-28.

Irwin, W. (1936). Propaganda and the News: Or, What Makes You Think So? Nueva York: Whittlesey House. 
Jansen, S. C. (2009). Phantom Conflict: Lippmann, Dewey, and the Fate of the Public in Modern Society. Communication and Critical/Cultural Studies, 6 (3), 221-245. Disponible en: https://doi.org/10.1080/14791420903049751.

Jowett, G. S. y O’Donnell, V. (2014). Propaganda and Persuasion. Los Angeles: Sage.

Kennedy, D. M. (1980). Over here: The First World War and America Society. New York: Oxford University Press.

Kidd, B. (1919). The Science of Power. London: Methuen \& Company.

Larrabee, H. A. (1920). The Formation of Public Opinion Through Motion Pictures. Religious Education, 15 (3), 144-154. Disponible en: https://doi.org/10.1080/0034408200150303.

Lasswell, H. D. (1927). Propaganda Technique in the World War. Nueva York: Alfred A. Knopf.

— (1941). Democracy through Public Opinion. Menasha, WI: George Banta Publishing Company.

Lee, I. (1925). Publicity: Some of the Things It Is and Is Not. New York: Industries Publishing.

Lincoln, C. A. (1920). Military Manpower: Psychology as Applied to the Training of Men and the increase of their effectiveness. New York: E. P. Button and Company.

Lippmann, W. (1922). Public Opinion. New York: Harcourt, Brace and Company.

Manz, W. H. (ed.) (2007). Civil Liberties in Wartime: Legislative Histories of the Espionage Act of 1917 and the Sedition Act of 1918 (2 vols.). Buffalo, N.Y.: W.S. Hein.

Marks, B. A. (1957). The Idea of Propaganda in America [tesis doctoral]. University of Minnesota.

Martin, E. D. (1920). The Behavior of Crowds: A Psychological Study. New York: Harper andBrothers. Disponible en: https://doi.org/10.1037/10905-000.

McDougall, W. (1920). The Group Mind: A Sketch of the Principles of Collective Psychology, with some Attempt to Apply them to the Interpretation of National Life and Character. New York and London: G.P. Putnam's Sons.

Merriam, C. E. (1919). American Publicity in Italy. American Political Science Review, 13 (4), 541-555. Disponible en: https://doi.org/10.2307/1944209.

Messinger, G. S. (2012). Battle for the Mind: War and Peace in the Era of Mass Communication. Amherst: University of Massachusetts Press.

Migliucci, D. (2016). Intolerable, peligrosa, imprescindible: intelectuales y políticos estadounidenses ante la problemática de la propaganda en el periodo de entreguerras (19191939). Rubrica Contemporanea, 5 (10), 45-64.

Monzón Arribas, C. (1987). La opinión pública: teorías, concepto y métodos. Madrid: Tecnos.

Moscovici, S. (1985). The Age of the Crowd: A Historical Treatise on Mass Psychology. Cambridge [etc.]: University Press.

Munson, E. L. (1921). The Management of Men: A Handbook on the Systematic Development of Morale and the Control of Human Behavior. New York: H. Holt.

Muñoz Alonso, A., Monzón, C., Rospir, J. I. y Dader, J. L. (1990). Opinion pública y comunicación política. Madrid: Eudema, D.L.

National Industrial Conference Board (1919). A Case of Federal Propaganda in our Public Schools: Some Criticisms of 'Lessons in Community and National Life', Issued by the United States Bureau of Education. Boston, Mass.: National industrial Conference Board, 1919. 
Park, R. E. (1915). The City: Suggestions for the Investigation of Human Behavior in the City Environment. American Journal of Sociology, 20 (5), 577-612. Disponible en: https://doi.org/10.1086/212433.

- (1923). The Natural History of the Newspaper. American Journal of Sociology, 29 (3), 273-289. Disponible en: https://doi.org/10.1086/213596.

- y Burgess, E. W. (1921). Introduction to the Science of Sociology. Chicago, Illinois: The University of Chicago Press.

Perry, E. (1920). Anti-American Propaganda in Hispanic America. The Hispanic American Historical Review, 3 (1), 17-40. Disponible en: https://doi.org/10.2307/2505841.

Ponsonby, A. (1928). Falsehood in Wartime. London: Allen and Unwin.

Read, M. J. (1941). Atrocity Propaganda: 1914-1919. New Haven: Yale University Press.

Religious Education (1919). The Twenty-First Meeting of the Council. Religious Education, 14 (6), 408-409.

Rodríguez Infiesta, V. (2016). Entre el bourrage de crâne y la sombra germánica: propaganda y comunicación social en Francia a través del discurso periodístico. Rubrica Contemporanea, 5 (10), 5-23.

Rospir, J. I. (2010). Opinión Pública: La Tradición Americana, 1908-1965. Madrid: Biblioteca Nueva.

Ross, S. H. (1996). Propaganda for War: How the United States was Conditioned to Fight the Great War of 1914-1918. London: McFarland.

Schrader, F. F. (1920). 1683-1920. New York: Concord Publishing Company Incorporated.

Sproule, M. J. (1997). Propaganda and Democracy: The American Experience of Media and Mass Persuasion. Cambridge-New York: Cambridge University Press.

Strong, E. K. (1922). Control of Propaganda as a Psychological Problem. The Scientific Monthly, 14 (3), 234-252.

Trotter, W. (1916). Instincts of the Herd in Peace and War. London: T. F. Unwin Ltd.

United States War Dept. General Staff (1919). Propaganda in its Military and Legal Aspects. Washington: Military Intelligence Branch, Executive Division.

Vaughn, S. (1980). Holding Fast the Inner Lines: Democracy, Nationalism, and the Committee on Public Information. Chapel Hill: University of North Carolina Press. 